\section{rev Psi}

Revista de Psicología (UNLP)

https://revistas.unlp.edu.ar/revpsi

\title{
El atravesamiento del fantasma, un acto que posibilita el advenimiento de la identidad sinthomática y de un lazo social no segregativo
}

\author{
Daiana Ballesteros $\quad$ Pablo González ${ }^{1,3}$
}

\section{Correspondencia}

dai_797@hotmail.com

Filiaciones institucionales

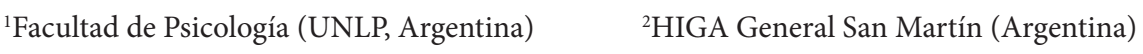

${ }^{3}$ HZGA Larrain (Argentina)

\section{Resumen}

Cuatro años después de su excomunión, Lacan propone una teoría del fin de análisis en La proposición del 9 de octubre de 1967 sobre el psicoanalista de la Escuela bajo la forma del atravesamiento del fantasma. Allí sostiene que en el pase lógico un analizante deviene psicoanalista y que, en ese mismo movimiento, se produce la caída del Otro con el cual el sujeto sostenía fantasmáticamente un lazo. Partiendo de esto, Bassols (2017) afirma que en La proposición Lacan interroga los fundamentos del vínculo social, en tanto se deduce de la misma que un análisis llevado hasta el final daría lugar a un modo de lazo particular, que no se sostendría en ninguna de las tres formas de la identificación freudiana. Este trabajo tiene por objetivo indagar el acto que da lugar al atravesamiento del fantasma y la nueva modalidad de lazo que se funda a partir de dicho movimiento.

\section{Palabras clave}

fantasma | acto | lazo social | segregación

Cómo citar

Ballesteros, D. y González, P. (2021). El atravesamiento del fantasma, un acto que posibilita el advenimiento de la identidad sinthomática y de un lazo social no segregativo. Revista de Psicología, 20(2), 167-183. HTTPS://DX.DOI.ORG/10.24215/ 2422572XE095

\begin{tabular}{lll}
\multicolumn{2}{l}{$\begin{array}{l}\text { Proceso editorial } \\
\text { Recibido }\end{array}$} & 1ra decisión \\
29 nov. 2020 & 23 feb. 2021 & $2422-572 \mathrm{X}$ \\
Aceptado & Publicado & Licencia \\
19 may. 2021 & 21 may. 2021 & $\begin{array}{l}\text { Licencia de Cultura Libre CC-BY 4.0 } \\
\text { (Compartir - Adaptar - Atribuir) }\end{array}$ \\
& & Entidad editora \\
& & RevPsi es una publicación de la \\
& & Facultad de Psicología (Universidad \\
& Nacional de La Plata, Argentina)
\end{tabular}




\section{A travessia do fantasma, um ato que possibilita o advento da identidade sinthomática e de um laço social não segregativo}

\section{Resumo}

Lacan, quatro anos depois da sua excomunhão, propõe uma teoria do fim de análise na Proposição de 9 de outubro de 1967 sobre o psicanalista da Escola sob a forma da travessia do fantasma. Ali sustenta que no passe lógico um analisante se torna psicanalista e que, nesse mesmo movimento, se produz a queda do Outro no qual o sujeito sustentava fantasmaticamente um laço. Partindo disso, Bassols (2017) afirma que na Proposição, Lacan interroga os fundamentos do vínculo social, no sentido de que deduz da mesma que uma análise levada até o final daria lugar a um modo de laço particular, que não se sustentaria em nenhuma das três formas da identificação freudiana. Este trabalho tem por objetivo indagar o ato que dá lugar a travessia do fantasma e a nova modalidade de laço que se funda a partir de dito movimento.

\section{Palavras-chave}

fantasma | ato | laço social | segregação

\section{The phantom crossing, an act that enables the advent of the sinthomatic identity and a non-segregating social bond}

\section{Abstract}

Four years after his excommunication, Lacan proposes an end-of-analysis theory in Proposition of October 9th, 1967 as understood by the psychoanalyst of the School in the form of the phantom crossing. There he argues that in the logical pass an analysand becomes a psychoanalyst, producing with such movement the fall of the Other with whom the subject phantomatically kept a bond. Based on this, Bassols (2017) states that in the Proposition, Lacan interrogates the basis of the social bond inasmuch as it may be deduced from it that an analysis conducted to the end would give place to a particular mode of bonding that would not be based on any of the three forms of Freudian identification. This work aims to explore the act that leads to the phantom crossing and the new bond modality resulting from such a movement.

\section{Keywords}

phantom $\mid$ act $\mid$ social bond $\mid$ segregation 


\section{La excomunión: un acto segregativo dentro de la comunidad analítica}

En su seminario Política lacaniana, Miller (2017) afirma que al utilizar este sintagma recurre a la historia para elevar algunos acontecimientos a principios susceptibles de constituir una política y estudiar la aplicación de los mismos en el presente y en el futuro. Es decir, no se trata de narrar acontecimientos, sino de extraer sus principios para hacer de ellos una orientación. En esta perspectiva, recorta una serie de hechos que han marcado una discontinuidad en la historia del psicoanálisis. A los fines de este artículo consideramos importante señalar uno de ellos: la excomunión de Lacan. Esto, con el objetivo de ubicar cómo la misma fue consecuencia de un acto segregativo impulsado por un fenómeno de grupo, así como los efectos que implicó para Lacan, quien luego de esto funda su Escuela y, con ella, un nuevo modo de lazo social entre aquellos que están implicados en la formación analítica.

La llamada excomunión es su expulsión de la IPA en 1963, a partir de la cual se le prohíbe para siempre tener la calificación de analista didacta, es decir, ser apto para formar analistas. En el procedimiento que antecede a dicha expulsión, Turquet realiza un informe donde formula:

Lacan es y seguirá siendo siempre inaceptable como didacta. Conviene perfeccionar garantías para su exclusión permanente. Toda tentativa de darle una categoría especial será desanimada y provocará un perjuicio desfavorable. Lacan como didacta es una amenaza [...] Es preciso un plan para mantener su exclusión de la enseñanza después de un eventual reconocimiento de la SFP. Que trabaje en paz y a su manera como simple miembro de la sociedad (Miller, 1987, p. 142).

Miller (2018) afirma que Lacan desdramatiza su excomunión en la medida en la que puede mostrar que lo que está en juego allí no es su propia persona sino el rechazo del concepto en psicoanálisis, el cual parece ser más intenso entre los analistas que en cualquier otro lugar. Respecto de este punto el autor considera importante distinguir los términos de intensión y extensión. Si concebimos al concepto como un conjunto, la extensión se refiere a los elementos que pertenecen al mismo, mientras que la pregunta de la intensión es de derecho e implica considerar las condiciones a las que deben responder los miembros para pertenecer a ese conjunto. En esta línea, partiendo del psicoanálisis puede decirse que:

la pregunta de la intensión de dicho concepto es ¿qué es el psicoanálisis? Y la que rápidamente se desprende de ella: ¿qué es un analista? El artículo indefinido responde justamente a que se lo toma como un analista cualquiera y, como la pregunta es de derecho, equivale estrictamente a ¿qué es el analista?, con el artículo definido (Miller, 2018, p. 48).

Entonces, si bien el psicoanálisis está en el mundo y existe como práctica, hay una 
incertidumbre sobre la intensión de dicho concepto, es decir, respecto de cuáles son las condiciones para nombrarse legítimamente psicoanalista. Es sobre este problema que Lacan se interroga y considera que su excomunión es el rechazo del grupo analítico de poner esto al trabajo.

Sus reflexiones lo llevan a afirmar que el psicoanálisis es una praxis en la cual se interviene lo real mediante lo simbólico y que, por lo tanto, hay un real en juego en la formación de los analistas. En este momento define a lo real como un agujero en el saber, como la barradura del Otro, que articula luego, en su enseñanza, al axioma "no hay relación sexual". Es decir, no hay norma que le permita al ser hablante saber qué hacer con el goce sexual y, en ese punto, cada uno tiene que inventar una solución sinthomática para arreglárselas con ello de un modo singular. Ahora bien, si partimos de ese real, el análisis no puede consistir en una reeducación emocional del paciente, ni en una adaptación del mismo a una normalidad ideal, así como tampoco puede tener como finalidad la identificación con el analista, como planteaba la IPA.

La formación del analista debe conducirlo a asumir ese real estructural y así dirigir la cura haciendo operar el deseo del analista. En el Seminario 11 Lacan sostiene que "el deseo del análisis es un deseo de obtener la diferencia absoluta" (Lacan, 2013, p. 284) entre el ideal y el objeto $a$. Este deseo se constituye como un principio de su política, que no trata de ajustar al sujeto a una realidad, sino que apunta a lo más singular, a que cada uno sea capaz de delimitar lo que lo diferencia como tal y de asumirlo. Se trata siempre de un deseo inhumano, no sólo en su renuncia a ocupar el lugar desde una concepción de poder, sino también en el no suponer la existencia de un bien que vale para todos.

Es por estas razones que Lacan propone, luego de su excomunión, una nueva versión de retorno a Freud que articula en su seminario de 1964 sobre Los cuatro conceptos fundamentales. Dicho retorno implica "esclarecer lo abrupto de lo real en el campo freudiano [...] El campo que Freud dejó a nuestro cuidado" (Lacan, 2016a, p. 247). Caracteriza a éste como un legado que hay que hacer fructificar, lo que significa también afirmar que Freud está muerto y eso puede constatarse en el hecho de que su creación, la IPA, se ha desorientado en cuanto a los principios que deberían guiar su acción.

Esto lleva a Lacan a cuestionar la política de Freud en su concepción originaria de la Internacional, política que se articula alrededor de los planteos que el autor hace en Tótem y tabú (1913/2012) con el lugar central que tiene allí la figura del padre. En esta línea, si partimos de considerar la respuesta que Freud da cuando se lo interroga respecto de qué pensaba de él mismo como analista, podemos ver que está advertido de la posición paterna que ocupa ante sus analizantes y alumnos. En este sentido afirma: "en la actualidad [...] tengo muchas cosas que me descalifican como gran analista. Una de ellas es que soy mucho el "padre" (Kardiner, 1979, p. 43).

Sobre este punto Miller (2017) sostiene que nosotros somos las consecuencias del fantasma de Freud, quien se ubica en el lugar del padre muerto y que el seminario de Los cuatro conceptos está concebido para exponer que el analista no es un padre, lo 
que implica, además, que el analista no es el gran Otro, sino que ocupa el lugar del objeto a. Asimismo, el autor sostiene que, si bien el análisis del padre es imposible, hay algo de éste que se deja descifrar en las encrucijadas del hijo.

$\mathrm{Al}$ analizar al hijo, precisamente, se ve la reconstrucción de la constelación que presidió su nacimiento, y esta constelación designa al padre. Por eso a partir de su excomunión Lacan percibe de qué modo ésta ya estaba programada por el padre. Es decir que Freud es objeto de un debate en la excomunión de Lacan por la IPA, en la medida en que esta última respondió a su deseo (Miller, 2018, p. 116).

Entonces, ir más allá del padre, supone ocuparse de su deseo y, más precisamente, de la causa de su deseo. Para Lacan (2013), hay algo en Freud que nunca fue analizado y, sin embargo, ese deseo opera desde el comienzo en la invención del psicoanálisis, en la apertura misma del campo freudiano. Es en este sentido que luego de su excomunión el autor -refugiado por la nobleza de sus amigos en la Escuela Normal Superior de París- decide no dictar el seminario que iba a llevar por título Los Nombres del Padre, sino ocuparse de los fundamentos del psicoanálisis. En este punto, se interroga respecto de qué lo autoriza a hablar de dichos fundamentos, considerando que le ha sido retirado el título de didacta en el que se autorizaba hasta el momento y que lo han echado de la institución de la que formó parte durante más de 20 años, sin posibilidad de regreso. Lacan podría haber denunciado las negociaciones que se hicieron respecto de él, caer en una posición reivindicativa, pero no lo hace. Toma la excomunión como un hecho y se pregunta en qué se autoriza para hablar, nada más ni nada menos que de los fundamentos del psicoanálisis, cuando ha ido más allá del padre y ya no cuenta con el Otro de la IPA, pero tampoco con el Otro universitario. Entonces, en la introducción a este seminario se plantea el problema de la autorización en el gran Otro. Es decir, desde qué lugar uno habla si se ha cortado el lazo con ese Otro simbólico que lo sostenía. El hecho de que él no denuncie eso como falsedad sino, que efectivamente se pregunte “ien qué me autorizo?" nos introduce en una lección ética que debe extraerse como enseñanza. En este sentido, afirma:

Como algunos saben, renuncié a esta función -a la que había de veras dedicado mi vida- debido a acontecimientos sucedidos dentro de lo que se llama una sociedad psicoanalítica, y justamente la misma que me había confiado dicha función. Se podría sostener que eso no pone en entredicho mi calificación para cumplir en otra parte esta función. Considero sin embargo este asunto como provisionalmente en suspenso. Y si hoy dispongo de los medios para poder, digamos solamente, dar continuación a esta enseñanza que fue la mía, se impone que, antes de abrir lo que se presenta entonces como una nueva etapa, comience por dar las gracias [...] (Lacan, 2013, p. 9). 
Que deje en suspenso la continuidad de su enseñanza forma parte, también, de una lección ética, ya que no se pasa de un Otro a la posición en la que él se encuentra sin un corte que sancione la discontinuidad. En esa introducción también se refiere a la excomunión como:

una censura nada ordinaria, puesto que se trata nada menos que de proscribir esta enseñanza, que ha de ser considerada como nula en todo lo tocante a la habilitación de un psicoanalista, y de convertir esta proscripción en condición para la filiación internacional de la sociedad psicoanalítica a la cual pertenezco (Lacan, 2013, p. 11).

O sea, que la condición para que la Sociedad Francesa de Psicoanálisis pase a ser de la IPA es que a Lacan se lo segregue, se lo haga desaparecer. Esa es la negociación. En la página 11 continúa diciendo:

Y esto aún no es suficiente. Está especificado que esta afiliación solo será aceptada si se dan las garantías de que mi enseñanza nunca podrá por medio de esta sociedad, entrar de nuevo en actividad para la formación de los analistas (Lacan, 2013, p. 11).

Entonces, cuando se refiere a la excomunión mayor, se refiere a la chammata, que es la imposibilidad de regreso. Lacan asume el real en juego en la formación, apartándose de las desviaciones que otros analistas sostienen respecto de la política y la ética de la cura fundadas por Freud. Elige siempre el psicoanálisis, su relación con el psicoanálisis, aún contra los analistas, y eso lo convierte en un hereje. Un hereje al que hay que segregar sin posibilidad de retorno. Se trata de producir su muerte simbólica, de proscribir la continuidad de su enseñanza y eso hace entrar al acto segregativo en una zona que Lacan había pensado como la segunda muerte. Eso, es la segregación absoluta.

\section{La Escuela y la proposición del pase: un acto en dos tiempos}

Miller (2018) sostiene que Lacan funda su Escuela -a la que define como "una experiencia inaugural"- en dos tiempos: en 1964 tenemos el acto de fundación, el cual es unilateral, en tanto dice "fundo" -y agrega- "tan solo como siempre he estado en mi relación con la causa analítica" (Lacan, 2016a, p. 247). En este primer momento Lacan considera que se puede desunir el psicoanálisis de la IPA y, si habla de una experiencia inaugural, es porque piensa que la misma no continúa a ninguna otra. Él asume allí una ruptura con lo que llamaba "la tradición continua desde los descubrimientos freudianos del psicoanálisis” y decide arreglárselas en forma diferente a la de Freud con los analistas -como resultado de un análisis- así como con la enseñanza y la transmisión. 
La hipótesis de Lacan es que Freud quiso sociedades ritualizadas y formalizadas para proteger al psicoanálisis de los psicoanalistas, asumiendo el riesgo de un estancamiento para que al menos sea preservado, incluso desconocido, el real en juego en el psicoanálisis. Es decir, las formas que Freud instituye esconden y hacen desconocer el real del que se trata, pero, al mismo tiempo, en cierta forma lo protegen. Miller (2017) subraya que resulta importante que Lacan haya señalado en la Proposición del pase (Lacan, 2016c) que es un hecho que Freud quiso dejar las sociedades analíticas tal cual son, ya que así admite que las formas de esas sociedades, de las que poco tiempo antes padeció una extrema censura, responden al deseo y al plan de Freud. Y es a partir de reconocer este hecho que intenta afirmar allí una experiencia inaugural, es decir, una experiencia de ruptura con la continuidad freudiana.

El segundo tiempo está marcado por La proposición del pase en 1967, documento que Lacan somete a discusión. Al respecto, Miller formula lo siguiente:

En el acto de fundación hay un agujero en el lugar del psicoanalista de la Escuela. Brilla por su ausencia algo que garantice la relación del sujeto con la formación recibida. En este vacío se elevará 3 años después la Proposición y hay que ver cómo se inscribe en la Escuela este texto que intenta definir la relación de garantía entre el sujeto y la formación que recibe [...] El pase y su Proposición son una propuesta a la Escuela sobre la base del Acto de fundación, que responde a la pregunta por la garantía que dicha Escuela puede aportar de que un sujeto, un analista, proviene de su formación (Miller, 2018, p. 209).

Los dos términos esenciales de la política que Lacan funda son la antinomia o el acuerdo que se debe encontrar entre el real en juego en la formación y los semblantes que los aparejan. Lacan cuestiona los semblantes de la Sociedad analítica, su texto Situación del psicoanálisis en 1956 (Lacan, 2014a) es explícitamente una sátira sobre los mismos.

El autor parte de la observación de que Freud crea la IPA diez años antes de trabajar en Psicología de las masas y análisis del yo, texto de1921, los mecanismos que operan para producir los lazos en la iglesia y el ejército. El descubrimiento fundamental de esas elaboraciones es considerar "la identificación del yo de cada individuo con una misma imagen ideal cuyo espejismo soporta la personalidad del jefe" (Lacan, 2014a, p. 446). Es este -para Lacan- el modo en que se organiza el lazo social en la IPA y afirma que en esta lógica la suficiencia de grado es el único grado en la jerarquía psicoanalítica. Se trata de una jerarquía que está más allá de toda prueba y que se transmite vía la reproducción imaginaria. Lacan sostiene que, si la entrada a la comunidad analítica implica como condición el psicoanálisis didáctico, entonces, hay alguna razón para que haya nacido en el círculo de los didactas la teoría que hace de la identificación con el analista el fin del análisis. Dos años más tarde afirma, en La dirección de la cura... (Lacan, 2014b) que esa razón supone el ejercicio de un poder. Es decir, que el semblante de didacta es usado -al interior de la IPA- para ejercer un poder sobre aquellos que están en formación. Es necesario aclarar que no 
hay en Lacan un desprecio de los semblantes como tales; para él hay lugares y funciones en la institución analítica que suponen encarnar necesariamente ciertos semblantes, pero se debe mantener una distancia de los mismos que posibilite usarlos para tratar e intervenir lo real y no para evitarlo.

Entonces, el primer principio que Miller extrae de estos acontecimientos es "no ceder ante el real en juego en la formación. Tal como Lacan lo entendió durante toda su vida, quiere decir no ceder ante los efectos transferenciales de su enseñanza: hay que asumirlos hasta el final" (Miller, 2017, p. 31).

Ahora bien, en 1968 un número notable de los alumnos de Lacan se van de la Escuela Freudiana de París que él había fundado en 1964, luego de que presentara su Proposición del 9 de Octubre de 1967 sobre el psicoanalista de la Escuela. En La razón de un fracaso (Miller, 2018) considera que dicho éxodo es efecto de que no logró despojar a sus alumnos del orden ceremonioso que sostienen: prefieren el semblante, el respeto de las formas, que considerar de qué se trata lo real. En la nota adjunta al Acta de fundación afirma que:

un psicoanalista es didacta, por haber hecho uno o varios psicoanálisis que demuestran ser didácticos. Se trata de una habilitación de hecho, que siempre se produjo así en realidad y que solo depende de un anuario que ratifica hechos... Se hace caducar el uso del consentimiento de los pares (Lacan, 2016a, p. 251).

El pase no es cuestionado como acontecimiento clínico, sino por el desplazamiento de fuerzas que produce, en tanto le devuelve el control de la Escuela a los no analistas, es decir, a los analizados (hasta el final), no a aquellos que pretendían ser analistas confirmados por la larga experiencia de su propia práctica. El fin de análisis pensado desde la perspectiva del atravesamiento del fantasma y la caída del objeto $a$, implica el desfallecimiento del sujeto supuesto saber y del Otro. El sujeto se encuentra -en el momento del atravesamiento- con la inexistencia del Otro; el orden simbólico a partir del cual ordenaba su mundo y daba sentido a su vida se le revela en su estatuto ficción y, en el mismo movimiento, cae la transferencia a ese Otro y al sujeto supuesto saber sostenido en el lazo con el analista. El analista queda entonces, en el fin del análisis, como resto de la operación, como un objeto que cae y la autoridad que encarnaba se desvanece.

Siguiendo estos argumentos, puede leerse que de esta concepción de final del análisis se desprende otro principio de política de Lacan: el analista solo se autoriza a partir de él mismo, en tanto una vez que se produce la caída del $a$ y el desvanecimiento del sujeto supuesto saber, no existe Otro en quien autorizarse. Surge así la problemática del ser analista. Esto no implica una cualidad, no es un título, sino que alguien accede a esa posición a partir del surgimiento del deseo del analista en la experiencia como analizante y no por el hecho de que practique el psicoanálisis. Es en este sentido que Lacan dice que "la terminación del psicoanálisis llamado en forma redundante didáctico es, en efecto, el paso del psicoanalizante a psicoanalista" (Lacan, 2016c, p. 
270). El AE es el que "desde entonces puede ir más allá, hasta volverse responsable del progreso de la Escuela, volverse psicoanalista de su experiencia misma" (Lacan, 2016c, p. 261). Es aquel que, habiendo terminado su análisis, puede testimoniar sobre los problemas cruciales en los puntos vivos donde están para el análisis, especialmente porque se encuentra en la tarea de resolverlos y es responsable del progreso de la Escuela.

En este punto, y retomando la primera clase de El seminario 11 y la pregunta que Lacan se hace allí sobre en qué autorizarse para hablar de los fundamentos del psicoanálisis, nos interesa destacar la dimensión cómica que él introduce, ya que consideramos que su posición en ese momento se articula a lo que plantea luego en su Proposición. En aquel pasaje afirma:

"La dimensión cómica no pertenece al registro de lo sucedido en la formulación de lo que llamé excomunión. Tiene que ver más bien con la posición en la que estuve durante dos años, la de saber que me estaban negociando; y me negociaban justamente quienes, respecto de mí, estaban en posición de colegas y hasta de alumnos [...] No quiero dejar la ocasión de señalar [...] que ello es, propiamente hablando, una cosa que puede vivirse, cuando se está adentro, en la dimensión de lo cómico. Creo que solo lo puede percibir plenamente un psicoanalista" (Lacan, 2013, p. 12).

Lo que Lacan plantea aquí es que en el mismo momento en que se le revela haber sido un objeto de negociación por parte de aquellos que integraban la institución que suponía para él un Otro simbólico, ese Otro cae y puede percibir, también, que ese Otro no existe. Ahora bien, él sostiene que solo alguien que ha advenido a la posición de analista puede encontrarse con esa dimensión de objeto que constituye al ser hablante, dimensión que a esta altura de su enseñanza responde al registro de lo real, tal como lo había anticipado en El seminario 10. Encontrarse con esa dimensión y percibir desde allí la inconsistencia del Otro, es lo que tres años más tarde, en la Proposición del 9 de octubre... formalizará como atravesamiento del fantasma. Podemos preguntarnos, entonces, si en ese momento de excomunión y el modo en que Lacan lo franquea, no hay también, un atravesamiento de su propio fantasma que hace que él advenga a la posición de analista, volviéndose psicoanalista de su experiencia y autorizándose en sí mismo para fundar una Escuela y una enseñanza.

\section{El acto analítico, problema central de la política del analista}

En La dirección de la cura y los principios de su poder, Lacan (2014b) introduce -junto a la estrategia y la táctica- una política del analista que lo invita a ubicarse por su falta en ser. Táctica y estrategia se ordenan en función de esta política que concierne a los fines de la acción y, por lo tanto, la cuestión del final del análisis depende de ella. Nos encontramos aquí con lo que el autor distingue más tarde como el acto analítico, problema central de la política del psicoanálisis. 
Es en esta línea que, al año siguiente de haber trabajado sobre La lógica del fantasma y un mes después de su Proposición del 9 de octubre del 67... donde habla del pase como fin del análisis, Lacan inicia un nuevo seminario bajo el título El acto psicoanalítico. Esta secuencia -que podemos sintetizar en fantasma-pase-acto-, como ya venimos anticipando, no es casual. En este apartado nos interesa establecer algunas precisiones respecto del concepto de acto y pensar a partir de esta noción, tanto la creación de la Escuela, como la interpretación analítica y la transformación que implica el final del análisis con el pasaje de psicoanalizante a psicoanalista.

El primer punto a destacar es que cuando hablamos de acto en el psicoanálisis de orientación lacaniana no nos referimos a un movimiento corporal, a meras acciones o comportamientos, ni a la simple puesta en marcha de la motricidad. Por el contrario, el acto tiene una íntima relación con las coordenadas significantes; consiste en atravesar un umbral, un límite simbólico y salir transformado de ese atravesamiento. Sabemos que el acontecimiento que Lacan elevó a paradigma del acto es el atravesamiento del Rubicón por parte de Julio César. Está claro que cruzar el Rubicón no conlleva ninguna dificultad. Si consideramos el comportamiento de César como un acto, es porque al cruzarlo va más allá del límite establecido por la ley romana y porque desde ese momento él se transforma, pasa automáticamente de aliado a enemigo de Roma y se desatan, a partir de ese acto, una serie de cruentas guerras civiles.

Podemos agregar también, siguiendo a Brodsky (2019), que el acto tiene la temporalidad del instante, del acontecimiento que no se repite y, en ese sentido, se opone al síntoma que no cesa de repetirse. Asimismo, un acto no puede considerarse la conclusión necesaria de una serie de premisas, ni tampoco la consecuencia inevitable de una cadena de causas y efectos. Cuando estamos ante un acto, hay siempre, entre las premisas y la conclusión, o entre las causas y el efecto, un hiato, una brecha, un salto. Es ese hiato el que hace del acto una decisión sostenida en la barradura del Otro, en la falla del saber, lo cual nos explica la cercanía entre el acto y la angustia. Con estas coordenadas podemos entender el deslizamiento significante que Lacan hace, en El seminario 15 (Lacan, 1967 - 1968), entre "acta" y "acto" al referirse a su acta/acto de fundación de la Escuela, en el año 64.

Los desarrollos de este seminario giran en torno a la conceptualización del acto psicoanalítico, respecto del cual Lacan sostiene que es sin Otro y sin sujeto. Es decir, el analista, en la dirección de la cura no es un sujeto dividido y no tiene un Otro en el cual garantizar sus interpretaciones. Enfocar el quehacer del analista a través de la perspectiva del acto implica hacer hincapié en las transformaciones que produce.

Asimismo, con acto psicoanalítico, Lacan no se refiere solo al acto del analista en la dirección de la cura, sino también -y esto nos interesa particularmente- al acto por el cual el analizante se transforma en analista. Es en este sentido que en la primera frase de la reseña del seminario escribe: "el acto psicoanalítico [...] he aquí que lo suponemos desde el momento electivo en que el psicoanalizante pasa a psicoanalista" (Lacan, 2016b, p. 395). Es conveniente poner en serie esta afirmación 
con la conocida -y ya aludida frase de la Proposición...- "el analista no se autoriza sino a sí mismo". Respecto de esto, Brodsky afirma que "toda la interrogación del Seminario 15 desemboca en interrogar al analista por su acto, no solamente en la dirección de la cura sino por ese acto a partir del cual es otro. Antes era analizante, paciente; ahora es analista" (Brodsky, 2019, p. 18).

Tomando los desarrollos que hace Lacan en los seminarios 14 y 15, se puede establecer una lógica de la cura pensada como un recorrido que tiene una posición inicial, que incluye una serie de transformaciones y que desemboca en una salida. Si seguimos la elucidación que hace Miller de esta lógica en su curso Donc (2011), podemos hacer la siguiente síntesis. La posición inicial del recorrido es localizada a partir de la negación del cogito cartesiano. Lacan sostiene que el pensar -se refiere aquí al pensar inconsciente- y el existir se oponen; entonces, la posición inicial es: no pienso y no soy. A partir de allí, hay que elegir: o pienso o soy. La elección "natural" del sujeto -a través de la alienación- es por la existencia: soy y no pienso. En esa posición es en la que generalmente llegan los pacientes a la consulta, afirmando una identidad yoica sostenida en identificaciones simbólicas y rechazando el pensamiento inconsciente. A través del operador transferencia, la entrada en análisis implica que el paciente pierde algo de su identidad y que comienza a asociar libremente -es decir, a pensar-, produciéndose la falta en ser y transformándose el paciente en un sujeto dividido. En esta nueva posición, entonces: pienso y no soy. Ahora bien, la gran pregunta que se hace Lacan en este momento es: ¿cómo se sale de esa indeterminación? Es decir, ¿cómo se termina un análisis?, ya que si nos quedamos en la falta en ser el análisis es interminable.

Sobre este punto nos interesa resaltar dos cuestiones. En primer lugar, que el autor señala, siguiendo la lógica de su cogito que, para salir de la falta en ser, es necesaria una nueva afirmación de identidad. Un nuevo "soy" que, obviamente, tiene que ser distinto al de la elección natural del soy pero no pienso, un "soy" que no pase por las identificaciones imaginarias y simbólicas, ni sea el "soy" del fantasma. Puede ubicarse aquí el "soy psicoanalista", siempre y cuando resaltemos la paradoja que implica esta frase, ya que ser psicoanalista no constituye una identificación. En segundo lugar, si Lacan habla del final de análisis como pase es porque lo plantea como la solución a un impasse; se trata de salir del rechazo del concepto en el que había caído el grupo analítico. Con el dispositivo del pase Lacan subvierte las relaciones de poder que se habían instituido en la IPA, ya que alguien deviene analista a partir de acceder a una posición sostenida en un deseo, la cual es producto de su trabajo como analizante. $\mathrm{Y}$ quienes reconocen que se ha a accedido a dicha posición no son quienes están en una relación de autoridad sino, más bien, aquellos que aún son analizantes, es decir, trabajadores.

Como se ha desarrollado previamente, en el momento del atravesamiento del fantasma se produce la caída del Otro y, aquel que deviene analista, encarna el objeto que es producto de la experiencia. Asumir esa dimensión de objeto -propia de todo ser hablante- y la ausencia de un Otro que de sentido a la existencia y opere como 
garantía de autorización, implica asumir lo real en juego en el psicoanálisis. Solo desde esta posición podrá tener lugar el acto analítico y solo en ese acto -y en las transformaciones que produce- alguien podrá nombrarse como psicoanalista.

Lacan reconoce y asume -luego de la excomunión- su posición de objeto y afirma "creo que solo lo puede percibir plenamente un psicoanalista" (Lacan, 2013, p. 12). Es decir, sostiene que si puede percibirlo es porque ha devenido psicoanalista. Consideramos que es desde esa posición que se autoriza de sí mismo y da lugar al acto de fundar su Escuela. Una Escuela de trabajadores donde no se rechace lo real en juego en la formación.

El final del análisis como atravesamiento del fantasma: la identidad sinthomática y el advenimiento de un lazo no segregativo

Bassols (2017) afirma que la Proposición... es un texto en el que Lacan cuestiona la identificación con el analista e interroga en sus fundamentos, la naturaleza del vínculo social, al preguntarse si es posible un lazo fundado en otro principio que el de las tres identificaciones freudianas clásicas: la identificación primaria con el padre, la identificación regresiva con el objeto de amor y la identificación histérica con el síntoma del otro. Como ya hemos mencionado, el sujeto del inconsciente, en tanto se define por ser un sujeto dividido, supone una identidad vacía, razón por la cual recurre a las identificaciones que le permiten decir "sé quién soy y dónde estoy", fijando su ser en el fantasma. De esta manera, estar identificado no solo supone ser sujeto del inconciente, sino también ser sujeto del grupo.

Ahora bien, todo análisis implica, en sus comienzos, una vacilación de las identificaciones y, en su final, se espera la caída de éstas, lo cual deja al descubierto la falta en ser, más allá de la ventana del fantasma. Es en ese momento de atravesamiento donde el sujeto puede ver qué era él, como objeto, en el deseo del Otro, así como también que ese Otro simbólico no existe realmente, sino que ha sido una suposición necesaria.

El autor plantea que la noción de caída de las identificaciones debe reformularse a partir de que se introduce -en la última enseñanza de Lacan- la identificación con el sinthoma. Desde esta perspectiva, no se trata de la desaparición de las identificaciones, sino de la reducción de sus efectos imaginarios, de separarse de ellas y desinvestirlas para poder servirse de las mismas, ya que no hay sujeto que pueda sostenerse sin identificación, tampoco, en la experiencia del pase. Es a partir de esto que afirma:

"Hay una identificación lacaniana del analista, que no es una identificación con el analista sino sin él, sin la definición de su identidad. Es una identificación que pasa por lo imposible, por lo imposible de decir, incluso de soportar, que debe pasar todas las veces que haga falta, para aislar ese real con el que hay que saber hacer cada vez en el grupo analítico. No ceder frente al propio deseo quiere decir para mí, no ceder nunca frente a este real en el que se funda el grupo analítico. Es a la vez no obviarlo, no dejarlo por imposible, sino tomarlo decididamente por imposible” (Bassols, 2017, p. 9). 
Entonces, si partimos de que no hay identificación posible de "el analista" como un conjunto de rasgos que lo definan de una manera universal, si el analizante ha dejado caer las identificaciones y lo que hay es un deser, la Escuela se constituye como un lugar necesario de elaboración sobre esta imposible identificación, la cual es diferente a las tres conocidas desde Freud, porque es sin Otro, lo cual implica identificarse a lo singular, a lo que no tiene parecido ni comparación con nada. Y si bien esto es imposible en el sentido lógico,

\footnotetext{
"la identidad sinthomática sería precisamente este intento [...] identificarse con lo más singular (sinthome), lo cual implica dejar de esperar que el Otro sea como uno mismo, lo que es a la vez el mejor seguro contra toda segregación o forma de racismo" (Bassols, 2017, p. 8).
}

Si seguimos esta línea argumentativa, podemos entender lo que plantea Lacan al final de su enseñanza, cuando sostiene que su anhelo es "la identificación con el grupo". Si bien esto puede parecer una contradicción visto desde la perspectiva de un sujeto desidentificado al final del análisis, en la perspectiva de la singularidad, de la identidad sinthomática, admite otras lecturas, si consideramos lo crucial de su planteo: "pero con esto no digo con qué punto del grupo tienen que identificarse" (Lacan, 1974-1975). Miller (2018) sostiene que, para avanzar en este punto, "hay que distinguir entre grupo y lazo social, en tanto el discurso analítico funda un lazo sin grupo y, en particular, sin obscenidad imaginaria" (p. 261). Es en esta línea que el autor ubica una paradoja: la Escuela funciona como un conjunto de Russell, es decir, como "el conjunto de los que no pertenecen a ningún conjunto". Esto implica, en términos lógicos, un conjunto que no sea segregativo, así como entender a la Escuela como la comunidad de aquellos que no tienen comunidad. Bassols (2017) deduce de estos argumentos la función del "más uno" como aquello que posibilita un vínculo social sin una identificación en el grupo. Es decir, no se trata de identificarse a una función imaginaria que genere consistencia a partir del reconocimiento mutuo de sus miembros, ni con la función simbólica del Ideal del Yo, sino más bien con una función "que debería saber hacer aparecer lo real en el que se funda el grupo, para hacer de él su brújula y saber tratar los espejismos de lo imaginario y los impasses de lo simbólico" (Bassols, 2017, p. 10).

Asimismo, Lacan propone -en su última enseñanza- dos identificaciones para el final del análisis: la del santo y la del trabajador. Nos interesa destacar esta última y su articulación con la transferencia de trabajo que, para Miller, está en el corazón del final del análisis, en tanto va a contracorriente del cinismo analítico y nos permite entender por qué un sujeto que ha llegado hasta este punto y ha verificado el deseo del analista en el final querría permanecer en el psicoanálisis dirigiendo curas y transmitiendo una enseñanza.

Lacan (2016a) introduce dicho término en el momento de la fundación de su Escuela, cuando sostiene que "la enseñanza del psicoanálisis no puede transmitirse de un sujeto a otro sino por los caminos de una transferencia de trabajo" (p. 254), lo 
cual implica que se trata de una enseñanza que se funda en la relación de un sujeto con otro, es decir, que no puede transmitirse de uno a todos, y que establece el relevo de su propio trabajo. Asimismo, en el Acto de fundación afirma lo siguiente: "los únicos callejones sin salida que hay que tener en cuenta por mi posición en una Escuela como esta, a saber, aquellos que engendraría en su trabajo la inducción misma a la cual apunta mi enseñanza" (Lacan, 2016a, p. 256). En este sentido, define a la Escuela a partir de una relación con un trabajador que incite a otro a trabajar, y después a otro, y que eventualmente estos diferentes otros acepten transmitir algo a lo que entonces puede llamarse Escuela.

En el pase, si aquel que ha devenido psicoanalista se identifica al trabajador, es debido a lo que tiene que saber bajo la forma del trabajo, a lo que tiene que demostrar a partir de las pruebas. Mientras que la transferencia analítica se articula a lo que el Otro sabe y al saber que ex-siste en el inconciente, en la transferencia de trabajo se trata de saber que se hace con la barradura del Otro. Es decir, precisamente porque el Otro no sabe hay motivos para construir.

El AE no es un sujeto para quien la transferencia habría sido reconducida a cero, sino alguien que quiere hacer algo a partir de $\mathrm{S}(\mathrm{A} /$ ); es un sujeto que se vale de $\mathrm{S}(\mathrm{A} /)$ para un trabajo, sobre el fondo de su ignorancia y de que el Otro no sabe. Para eso, si la Escuela es lo que debe ser, se enlaza a ella. Consiguientemente, la causa analítica es un lazo indisoluble (Miller, 2018, p. 177).

El autor señala que el pase está más allá del amor al saber, lo que no significa que esté más allá del saber. En este punto marca una diferencia entre la enseñanza y la práctica del psicoanálisis, ya que, mientras el saber supuesto en esta última sirve de inducción al amor, en la enseñanza, debe inducir al trabajo. Ahora bien, es aquí donde introduce la siguiente idea, que nos interesa destacar:

cuando para un sujeto el saber ya no es inducción al amor, a la transferencia y tampoco al trabajo, puede ocurrir que el saber sea inducción al odio. Luego, cuando por un lado el lazo analítico está terminado, y por otro no se tiene acceso a este más allá y se permanece en grupitos que se multiplican, creo que en ese intervalo se forja el odio [...] Entonces, cuando siento venir o anunciarse un periodo de odio, propongo en su lugar, trabajo del saber a cielo abierto (Miller, 2018, p. 192)".

Entonces, si el grupo fundado en el rechazo del concepto puede dar lugar al odio y a actos segregativos como la excomunión de Lacan, asumir la función de más uno puede motivar, como sostiene Bassols (2017) “una verdadera acción política desde el psicoanálisis fuera de todo partidismo" (p. 10), la cual implicaría hacer de la excepción que es siempre cada sujeto en el grupo, de la singularidad de su síntoma, algo que valga para todos y para cada uno de ellos. 
"Producir un efecto así [...] es necesariamente ser herético de ese grupo. Un herético es el que se identifica con su sinthoma, de modo que no pueda hacerse con eso ninguna ortodoxia posible [...] La Escuela del pase es la Escuela de una transferencia de trabajo constante sobre este punto, para poder establecer un nuevo lazo social fundado en lo herético de la identificación con el sinthome" (Bassols, 2017, p. 10).

Consideramos que Lacan intentó ubicarse como un herético respecto de la IPA, de sus rituales y de su organización en torno al padre muerto, que renegaba del real que está en juego en la formación del analista, pero no logró producir el efecto que terminaría de constituirlo en esa posición, y como consecuencia de sus interpretaciones tuvo lugar la excomunión. Y si bien la IPA estaba organizada de tal modo que se presentaba refractaria a las mismas, no podemos desconocer que también Lacan seguía ligado al padre, sin poder ir más allá de él. Quizá era este lazo fantasmático, que todavía no se había conmovido, lo que le impidió advenir, en ese momento, a la posición de un herético decidido.

\section{Conclusiones}

En el presente trabajo hemos realizado un recorrido partiendo de algunos hechos que han marcado una discontinuidad en la historia del psicoanálisis y de los cuales se pueden extraer principios para una política que oriente nuestra acción. Respecto del primero de ellos, la excomunión de Lacan de la IPA en 1963, consideramos que la misma es efecto de un fenómeno de grupo que se articula al modo en que Freud organizó a la comunidad analítica -siguiendo los lineamientos de Tótem y tabúalrededor de la figura ideal del padre muerto. En este sentido, los lazos se sostienen sobre la consistencia imaginaria que produce el reconocimiento mutuo de sus miembros, articulados a la dimensión simbólica del Ideal del Yo. Y es en la medida en que Lacan busca reintroducir el real que está en juego en la formación analítica, que se produce el fenómeno segregativo que implica, no solo su expulsión de la IPA, sino la proscripción absoluta de su enseñanza.

A partir del modo en que Lacan atraviesa este punto, extraemos varias lecciones éticas. En primer lugar, no asume una posición reivindicativa, sino que toma la excomunión como un hecho que articula al rechazo del concepto en psicoanálisis. Es decir, lee que se trata de un fenómeno de estructura en el marco de cómo estaba organizada la Internacional en ese momento, y cómo esto responde al plan y al deseo de Freud para preservar lo real en juego en el psicoanálisis. Por otro lado, deja en suspenso la continuidad de su enseñanza y se pregunta en qué se autoriza para hablar de los fundamentos del psicoanálisis, en la medida en que se ha producido la caída del gran Otro en que se sostenía.

Ahora bien, es justamente en este momento donde advierte también, el lugar de objeto de negociación en el que estuvo y, al mismo tiempo en que eso se revela, puede percibir también que el Otro no existe. Sobre esto planteamos el interrogante 
respecto de si la excomunión y el modo en que Lacan la atraviesa no implica, también, un atravesamiento de su propio fantasma que hace que él vaya más allá de Freud, adviniendo a la posición de analista y autorizándose en sí mismo para hacer un acto que hemos delimitado -siguiendo a Miller- en dos tiempos: el acto de fundar la Escuela y la Proposición del Pase donde plantea su primera teoría del fin de análisis como atravesamiento del fantasma.

En este punto el acto, problema crucial de la política del psicoanálisis, toma un nuevo relieve, al pensarse no solo en términos de la cura, sino también del acto que posibilita que el analizante se transforme y advenga a una nueva posición que le permite afirmar "soy psicoanalista" sin que eso suponga una nueva identificación. Respecto de esta cuestión, nos hemos valido de los desarrollos de Miller y Bassols, para explicar la paradoja que implica la imposible identificación del analista. Sobre esto, extraemos cuatro puntos cruciales: 1) Debido a que no hay sujeto que pueda sostenerse sin identificación, el atravesamiento del fantasma y la caída de las identificaciones que conlleva no debe entenderse como la eliminación de las mismas, sino como la reducción de sus efectos imaginarios; se trata de desinvestirlas para poder servirse de ellas. 2) Una vez atravesado el fantasma adviene una identificación que es sin Otro, que implica subjetivar que no hay el significante del analista e identificarse con lo más singular, con el sinthome y, desde allí, asumir la función de más uno, para interpretar a la Escuela y hacer surgir el real en el que se funda, deviniendo así, herético del grupo. 3) Lacan propone, como una identificación posible al final del análisis, la figura del trabajador. El AE es aquel que, sobre el fondo de su ignorancia y habiendo asumido que el Otro no sabe, sostiene un trabajo decidido de transmisión sobre lo real que está en juego en la formación del analista. 4) Este trabajo engendra una transferencia que induce a otros a trabajar y genera un lazo indisoluble ligado a la causa analítica. Asimismo, ese trabajo es un modo de tratar el odio que puede surgir cuando el saber ya no induce al amor y a la transferencia.

Por último, si un sujeto se constituye como herético como efecto de una interpretación que hace aparecer lo real del grupo y posibilita hacer de éste su brújula, podemos suponer que Lacan no logra acceder a esta posición mientras forma parte de la IPA, ya que sus intervenciones no producen efectos que permitan elevarlas al estatuto de una interpretación y, por lo tanto, de un acto analítico. Es luego de la excomunión que él puede ir más allá del padre y fundar su Escuela y su enseñanza. El modo en que él franquea este momento tiene la estructura del atravesamiento del fantasma: el Otro cae y el adviene objeto causa del trabajo en una comunidad fundada en lo real como imposible. Consideramos que es en ese momento donde Lacan se constituye como un herético decidido, un herético que, afirmado en su identidad sinthomática, funda una política contra toda segregación o forma de racismo. 


\section{Referencias} $\begin{array}{cc}\text { Bassols, M. (2017). La imposible identificación del } \\ \text { analista. } & \text { Recuperado de }\end{array}$

WWW. X XVIIJORNA D A S A N UA LES.C OM/ TEMPL ATE.PH P ? FIL E $=$ TEXTOS - D E ORIENTACION\%2FLA-IMPOSIBLE-IDENTIFICACIONDEL-ANALISTA.HTML

Brodsky, G. (2019). Fundamentos. El acto analítico. Cuadernos del ICdeBA.

Freud, S. (1913/2012). Tótem y tabú. En Obras Completas, Tomo XIII. Amorrortu.

Kardiner, A. (1979). Mi análisis con Freud. J. Mortiz.

Lacan, J. (1966-1967). El seminario, Libro XIV: La lógica del fantasma. Inédito.

Lacan, J. (1967-1968). El seminario, Libro XV: El acto psicoanalítico. Inédito.

Lacan, J. (1974-1975). El Seminario, Libro XXII: RSI Inédito.

Lacan, J. (2013). El Seminario, Libro XI: Los cuatro conceptos fundamentales del psicoanálisis. Paidós.

Lacan. J. (2014a). Situación del psicoanálisis y formación del psicoanalista en 1956. En Escritos I. Siglo XXI.
Lacan, J. (2014b). La dirección de la cura y los principios de su poder. En Escritos II. Siglo XXI.

Lacan, J. (2016a). Acto de fundación. En Otros escritos. Paidós.

Lacan, J. (2016b). El acto psicoanalítico. Reseña del seminario de 1967-1968. En Otros escritos. Paidós.

Lacan, J. (2016c). Proposición del 9 de octubre de 1967 sobre el psicoanalista de la Escuela. En Otros escritos. Paidós.

Miller, J-A. (1987). El informe de Turquet (19 de mayo de 1963). En Escisión- Excomunión- Disolución. Manantial.

Miller, J-A. (2011). Donc. La lógica de la cura. Paidós.

Miller, J-A. (2017). Política lacaniana. Colección Diva.

Miller, J-A. (2018). El banquete de los analistas. Paidós. 